\title{
Utility of 'dual phase' cone beam computed tomography during radioembolisation in patients with hepatocellular carcinoma: what is really changing in flow dynamics before and after ${ }^{90} \mathrm{Y}$ delivery?
}

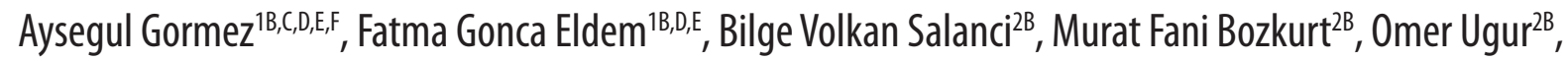 \\ Bora Peynircioglu ${ }^{1 A, B, D, E}$
}

'Department of Radiology, Faculty of Medicine, Hacettepe University, Turkey

${ }^{2}$ Department of Nuclear Medicine, Faculty of Medicine, Hacettepe University, Turkey

\section{Abstract}

Purpose: The aims of the study were: 1) to compare two phases of dual-phase cone beam computed tomography (DPCBCT) achieved before and after Yttrium-90 $\left({ }^{90} \mathrm{Y}\right)$ administration and to evaluate additional benefits during radioembolisation (RE) procedures; and 2) to compare DP-CBCT with pre-procedure contrast enhanced cross-sectional images in terms of tumour detection.

Material and methods: Twenty-three hepatocellular carcinoma patients undergoing RE treatment were scanned with DP$\mathrm{CBCT}$ consisting of early arterial (EA) and late arterial (LA) phases before and after ${ }^{90} \mathrm{Y}$ administration. The CT-like datasets were compared according to embolisation effect, enhancement patterns, lesion detectability, image quality, and artifacts by two interventional radiologists blinded to each other. The compatibility of the two radiologists was evaluated with kappa statistical analysis, and the difference between EA and LA phases was evaluated with marginal homogeneity test. Also, DP-CBCT images were compared with preprocedural cross-sectional images (CT/MRI).

Results: For 23 patients 92 data were acquired. Thirteen patients showed a decrease on post-embolisation images both visually and on Hounsfield unit (HU) measurements. No statistical difference was found for tumour detection between EA and LA phases $(p=1.0)$. Tumour enhancement was visually superior at LA phases whereas EA phases were better for arterial mapping for selective catheterisation. DP-CBCT images were not inferior to preprocedural cross-sectional imaging findings.

Conclusions: DP-CBCT is a promising tool for predicting tumour response to therapy and is not inferior to preprocedural cross-sectional imaging in terms of tumour detection. It allows better assessment during RE procedures because early phases provide good mapping for superselective catheterisation whereas late phases are better for visualisation of tumour enhancement.

Key words: hepatocellular carcinoma, radioembolisation, cone beam CT, Y-90.

\section{Introduction}

Hepatocellular carcinoma (HCC) is the most common primary liver cancer and the third most common cause of cancer death worldwide [1,2]. The prognosis is poor due to delayed diagnosis and is one of the most common mortality causes in patients with cirrhosis $[3,4]$. Patients with high tumour load and/or comorbidities that are not suitable for surgery are treated with different systemic treatment options or local-regional therapies to decrease tumour load and improve the patient's life quality and expectancy [5]. Radioembolisation (RE) is one of the optional regional therapies for inoperable HCC lesions where implantable radioactive microspheres

Correspondence address:

Dr. Aysegul Gormez, Department of Radiology, Faculty of Medicine, Hacettepe University, Turkey, e-mail: gundogdu.aysegul@gmail.com

Authors' contribution:

A Study design · B Data collection · C Statistical analysis · D Data interpretation · E Manuscript preparation · F Literature search · G Funds collection 
(Yttrium-90 $\left[{ }^{90} \mathrm{Y}\right]$ ) are delivered to the feeding arteries. Like other intra-arterial procedures, RE has been evaluated for patients who are not eligible for resection or ablation, mostly with larger infiltrating and/or multifocal nodular tumours [6].

Cone beam computed tomography $(\mathrm{CBCT})$ is a technology in which the $\mathrm{C}$ arm of the flat panel angiography rotates around the patient, acting as a CT detector providing data that can be reconstructed as cross-sectional CT images during interventional procedures [7]. The images acquired when the patient is on the table enable the interventional radiologist to detect lesions that cannot be seen with routine catheter angiography [8]. CBCT is now widely used during liver tumour embolisation to ensure super selective catheterisation, thus avoiding non target embolisation, and to enhance detection of tumours that cannot be seen with routine catheter angiography [9]. It can provide information for evaluating the extra hepatic leakage, parasitic feeders, portal vein invasion, and also for monitoring the embolisation effect [10]. One study showed improved survival rates in unresectable HCC patients when chemoembolisation is done with CBCT assistance [11]. In standard practice CBCT provides early arterial phase images. However, it has been shown that imaging of both early arterial (EA) and late arterial (LA) phases provides superior tumour detection than single-phase imaging $[8,12,13]$. So far, in the current English literature all studies of dual phase cone beam CT (DP-CBCT) have been made with transcatheter arterial chemoembolization (TACE) patient groups, and no study has ascertained whether DP-CBCT is a useful technology in detecting HCC nodules during RE. Therefore, in our study we aimed to demonstrate the feasibility of DP-CBCT with one contrast injection using a commercially available dual-run CBCT acquisition protocol software (syngo DynaCT, Siemens Healthcare) in HCC patients during RE. The purposes of the study were: 1) to compare two phases of DP-CBCT achieved before and after ${ }^{90} \mathrm{Y}$ administration and evaluate additional benefits during RE procedures; and 2) to compare DP-CBCT with pre-procedure contrast-enhanced cross-sectional images in terms of tumour detection.

\section{Material and methods}

This prospective study was approved by our institutional ethical committee, and informed consent was obtained from all patients. HCC patients referred to our interventional unit for RE treatment were first evaluated regarding their performance status and liver functions, and the ones staged as Barcelona-Clinic Liver Cancer (BCLC) stage B and $\mathrm{C}$ were found to be eligible for RE treatment. From these patients, the ones who were considered capable of cooperation for the required breath holding and could tolerate mild sedation were enrolled in the study.

\section{Patients}

There were a total of 23 either pathologically or radiologically diagnosed HCC patients (four women, 19 men), with a mean age of $64.9 \pm 5.75$ years (range $55-73$ ). The etiology was hepatitis B virus (HBV) in 12 patients, hepatitis $\mathrm{C}$ virus (HCV) in two patients, and the rest had idiopathic chronic parenchymal disease. In two patients extra hepatic involvement was seen (portocaval-periceliac lymphadenopathy). Ten patients had portal vein thrombosis (four total, six partial). Ten patients had undergone a previous treatment; Sorafenib treatment $(n=1)$, systemic chemotherapy $(n=2)$, chemoembolisation procedure $(n=4)$, resection $(n=2)$, and resection-radiofrequency ablation and chemoembolisation $(n=1)$.

\section{Radioembolisation}

After preprocedural evaluation of all laboratory and imaging findings, all patients first underwent angiographic assessment and Tc-99 labelled macro albumin aggregate (MAA) infusion for test angiography in which all necessary gastrointestinal feeders were embolised, followed by MAA infusion for determining extra hepatic lung shunts and feeders to the gastrointestinal system. All patients were imaged with scintigraphy and/or single-photon emission-computed tomography (SPECT) CT within one hour of the test angiography, and all of them were found to be eligible for the treatment. One patient showed extra hepatic leakage towards the umbilicus through the falciform ligament. He was not excluded from the study but received the treatment under ice compression.

Radioembolisation with ${ }^{90} \mathrm{Y}$ was performed within one month (mean $42.6 \pm 18$ days) of the MAA assessment. Treatment strategy of lobar or whole liver ${ }^{90} \mathrm{Y} \mathrm{RE}$ was determined according to the extent of disease. A catheter was placed into the same location as during the MAA procedure, and glass microspheres were used for ${ }^{90} \mathrm{Y}$ administration. For each patient DP-CBCT was performed before and after ${ }^{90} \mathrm{Y}$ infusion. Thus, out of 23 patients a total of 92 DP-CBCT datasets were gathered. After the procedure Bremsstrahlung imaging was performed within one hour to prove the absence of extra-hepatic leakage. All patients were discharged the following day with normal liver function. Laboratory and cross-sectional imaging follow-up was done after one week, one month, and three months.

\section{Dual-phase cone-beam computed tomography technique}

Dual-phase cone-beam CT imaging was performed on a flat-detector angiographic system (Artis zee Biplane, Siemens Healthcare, Forchheim, Germany), which enabled CBCT acquisition and volumetric image reconstruction (Feldkamp back projection) according to the injection and 


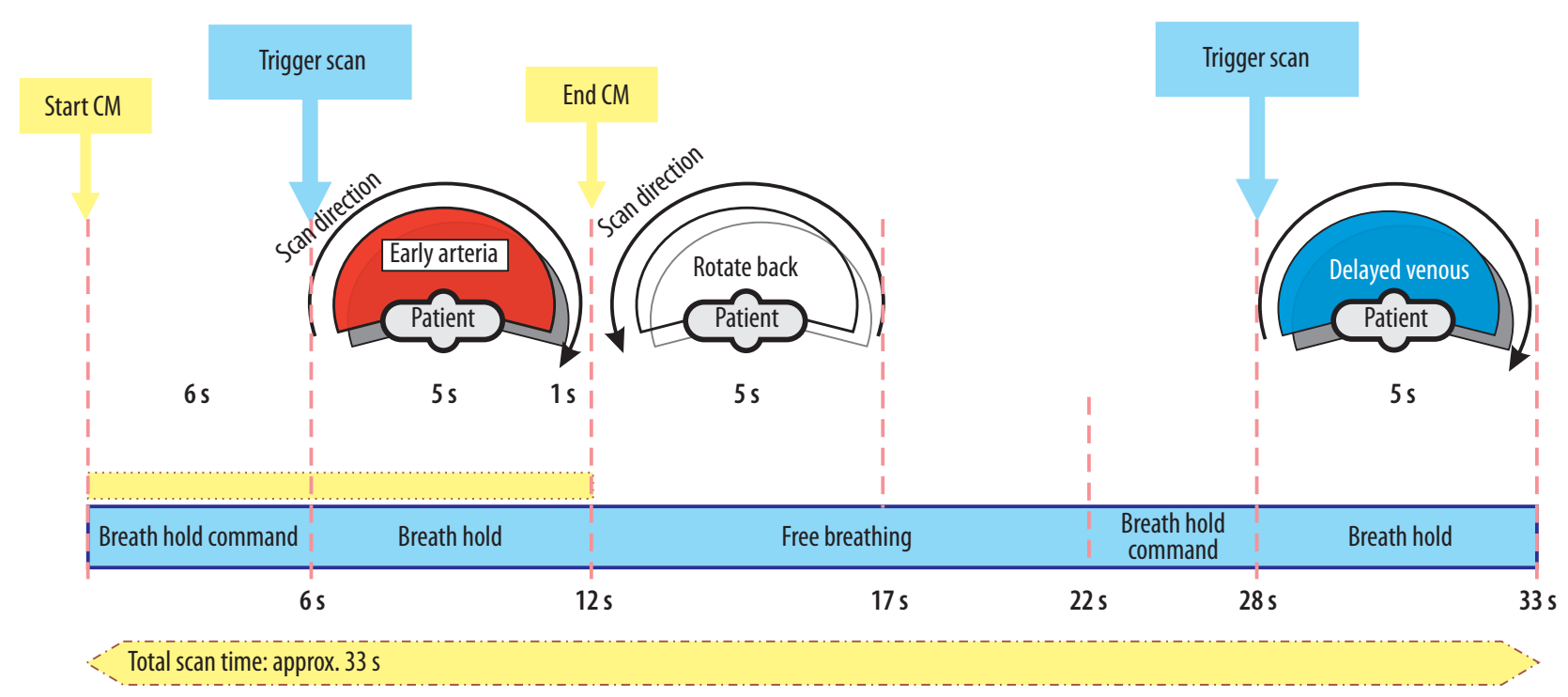

Figure 1. The diagram showing the timing of the breath hold command and contrast injections in dual-phase C arm computed tomography technique

acquisition protocol shown in Figure 1. The DP-CBCT acquisition consisted of two runs: an initial rotation that captured the EA phase followed by a second rotation that captured the LA phase. The following parameters were used in data acquisition: acquisition time for each rotation approximately $5 \mathrm{~s}, 90 \mathrm{kV}$ set $\mathrm{kVp}$, detector entrance dose $0.36 \mu \mathrm{Gy} /$ frame, $616 \times 480$ image matrix, projection on $30 \times 40 \mathrm{~cm}$ flat panel, $200^{\circ}$ total angle, $0.8^{\circ}$ angle increment/frame, and 248 frames in total.

A total volume of $36 \mathrm{ml}$ of $25 \%$ diluted iodinated contrast medium (Ultravist-300, Bayer-Schering Pharma, Berlin, Germany) was injected through the $2.8 \mathrm{~F}$ coaxial microcatheter into the hepatic artery at a rate of $3 \mathrm{ml} / \mathrm{s}$ using a power injector (Medrad, Indianola, PA, USA) at 300 psi for each DP-CBCT acquisition.

Images were obtained as EA and LA phases respectively at $6 \mathrm{~s}$ and $28 \mathrm{~s}$ with two breath holds (Figure 1). The projection data of each acquisition run was sent automatically to a dedicated workstation (syngo XWP, Siemens Healthcare, Forchheim, Germany) on which CT-like axial slices with an isotropic voxel size of $0.5 \mathrm{~mm}$ were reconstructed for each phase. DP-CBCT imaging was performed for all 23 patients pre- and post-embolisation, resulting in 46 pairs of EA and LA phase images. These 92 reconstructed CBCT series were evaluated using Multi Planar Reformat (MPR) or Maximum Intensity Projection (MIP) side by side.

\section{Image analysis}

Images were evaluated by two interventional radiologists (BP, 15 years and GE, five years of IR experience) blinded to each other. They were asked to classify and compare preand post-embolisation images regarding the following:
1. Embolisation effect: Although RE is less embolic comparing TACE, pre- and post-embolisation images were evaluated both visually and by Hounsfield unit (HU) measurements to see if there were any embolisation effects that could be detected on tumour enhancement. Targeting a single lesion for multifocal tumours, and lobar and segmental lesions for each patient, pre-embolisation EA images were compared with post-embolisation EA images, and pre-embolisation LA images were compared with post-embolisation LA images.

2. Enhancement patterns: Pre-embolisation images were compared with basal cross-sectional images (CT/MRI) regarding tumoural contrast enhancement. Also, the two phases themselves were compared.

3. Lesion detectability: Comparison of each phase (EA and LA) of pre-embolisation images with preprocedural cross sectional images (CT/MRI) as well as the two phases with themselves was performed regarding lesion detectability. Lesions were categorised as diffuse lobar, segmental, and multifocal. For infiltrative tumours lobar expansion and for multifocal tumours number of tumours were noted.

4. Image quality: Pre-embolisation EA and LA phase CBCT images were evaluated for image quality and scaled as optimal (2 points), diagnostic (1 point), or non-diagnostic (0 point).

5. Artifacts: Breath artifacts were assessed visually and also objectively by measuring maximum diaphragm movement in millimetres and its peak time on sagittal plane from the raw data on pre-embolisation and post-embolisation early and late phases. Other artifacts that affected the image quality such as coils, lumen in the contrast, metallic artifacts, etc. were also noted. 


\section{Statistical analysis}

The compatibility of the two radiologists was evaluated with kappa statistical analysis for each parameter. The differences between early and late arterial phases was evaluated with marginal homogeneity test.

\section{Results}

All 23 patients were successfully treated with RE without any complications, and the procedural success rate was $100 \%$. Eighteen patients received right lobar treatment, two patients received left lobar treatment, and three patients received whole liver treatment. Technical success for DP-CBCT was $100 \%$. Performing a DP-CBCT acquisition pre- and post-radioembolisation in 23 patients, a total of 92 CBCT datasets were acquired with dual-phase protocol both EA and LA phases (Figures 2 and 3) were performed with a single intra-arterial contrast infusion.

\section{Embolisation effect}

Out of 23 patients, 21 were evaluated for embolisation effect. Two patients were excluded from this evaluation due to catheter displacement. Looking at two image series before and after embolisation, the following changes were noted visually and quantitatively. Thirteen patients showed a decrease on post-embolisation images both visually and on HU measurements. Four patients showed an increase in HU measurements. Four patients showed no difference visually between pre- and post-embolisation images. Of note, four total and four partial portal vein occlusions were found in these eight patients. Although visual changes were noted, the interobserver agreement was considered to be low, being $68 \%$ on early arterial phases and $35 \%$ on late arterial phases. No difference was found between early arterial and late arterial phases on the marginal homogeneity test within the image interpretation of the two radiologists.
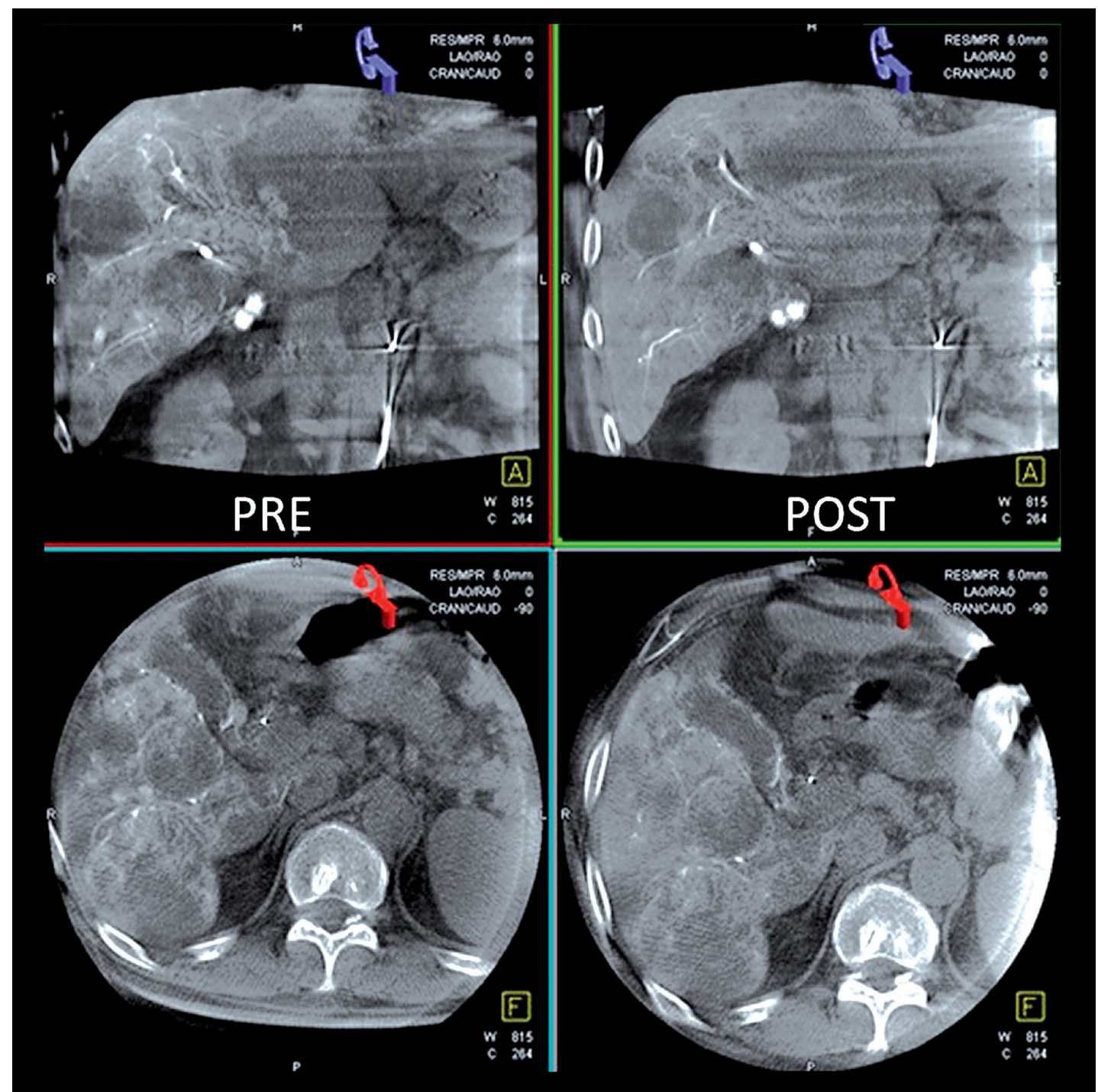

Figure 2. Pre- and post-embolisation axial and coronal early arterial phase images 


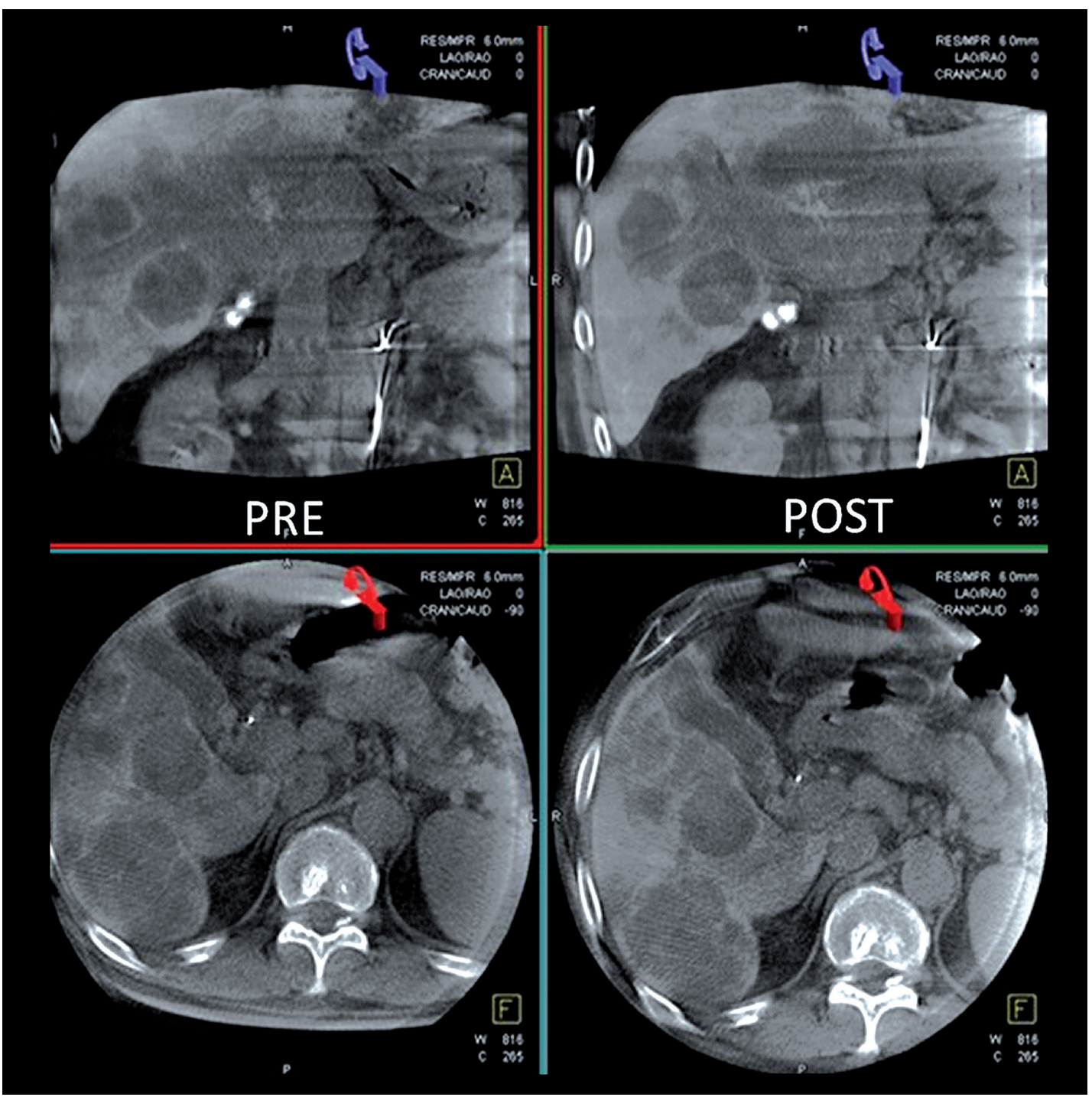

Figure 3. Pre- and post-embolisation axial and coronal late arterial phase images

\section{Enhancement patterns}

Both radiologists agreed that EA phases contrast enhancement in the vessel lumen was prominent with a $100 \%$ consistency in showing an arterial feeder mapping. Tumour enhancement was visually superior at LA phase to EA phase. When compared with cross-sectional images, dual-phase C-arm CT images were not found to be inferior regarding tumour enhancement except for one patient with a portal vein tumour, in whom cross-sectional images were found to be prominently superior in tumour enhancement. Consistency between two radiologists was $100 \%$. Of note, none of the tumours showed an area lacking enhancement that would be suspicious of an extrahepatic feeder.

\section{Lesion detectability}

In ten patients - lobar involvement, in three patients segmental involvement, and in nine patients - multifocal involvement was seen. One patient had a tumour in the portal vein lumen. All of the lesions' characteristics were found to be the same with the preprocedural CT by both radiologists (sensitivity of lesion detection was $100 \%$ ). According to lesion detectability, the two radiologists' compatibility was $100 \%$. No difference was found in the marginal homogeneity test between EA and LA phases $(p=1.0)$.

\section{Image quality}

Pre-embolisation EA and LA phases were scored by two radiologists according to image quality, and the scores were summed as optimal ( 2 points), diagnostic ( 1 point), or non-diagnostic ( 0 point). Out of a total maximum score of 92 for 23 patients, EA phases total score was 69 and LA phases score was 73, with a consistency between two radiologists of 96: 6\% for early phase and $96 \%$ for delayed phases. The main factors affecting the image quality were patient arm positioning, obesity, and suboptimal patient positioning, among the ones scored as poor image quality. 


\section{Artifacts}

Measured from the raw data, the mean value of diaphragm motion was $5.4 \mathrm{~mm}(0-27 \mathrm{~mm})$ on pre-embolisation images and $3.28 \mathrm{~mm}(0-19 \mathrm{~mm})$ on post-embolisation images. The mean time of maximum motion was $0.8 \mathrm{~s}$ on pre-embolisation images and $0.6 \mathrm{~s}$ on post-embolisation images showing discordancy with the breath hold command given at the beginning of the scan. On visual assessment the consistency between the two radiologists was $86.2 \%$ for early phases and $88.1 \%$ for delayed phases. No statistical difference was found between the two phases, and no accordance was found between visual and objective assessments statistically $(p<0.05)$ (first observer $k=-0.037$ and second observer $k=-0.189$ ).

All patients showed streak artifacts on early arterial phase images related to hepatic segmental vessels being filled with contrast, although the contrast used was diluted with saline.

Other artifacts seen affecting the image quality were streak artifacts, caused by metallic clips and coils.

\section{Discussion}

In the literature, DP-CBCT was used for RE patients in only three studies previously. One of them was in a neuroendocrine patient group, one of them was with metastatic disease, and the third was a case-report. Our study is the first one performed in HCC patients treated with $\mathrm{RE}$. In the literature most of the studies are focused on chemoembolisation patient groups. Thus, these are the first outcomes of HCC patients treated with RE.

In HCC, tumour enhancement is known to be an indicator of active disease and is accepted as one of the tumour response criteria [14]. This led us to compare the images of pre and post ${ }^{90} \mathrm{Y}$ infusion in terms of embolisation effect, although RE is known to be less embolic than TACE. This is the first study comparing these two couples of CBCT data achieved during RE with HCC patients. Previous studies done with TACE easily pointed out the post-embolisation effect as an expected outcome of the embolic nature of TACE $[15,16]$. There is only one RE study on DP-CBCT using quantitative measurement methods, which involved a liver metastasis patient group using arbitrary units (AU), in the English language literature [17]. This study showed decreasing results in AU after RE. In our study in 13 patients the decrease was shown visually and quantitatively after RE. Although some differences were noted on the measurements, the interobserver agreement on visual correlation is low. CBCT measurements may not be reproducible because HU measurements have not been validated like multidetector CT HU measurements. Therefore, it should be considered that these changes may not be reflecting the true decrease of the tumour contrast enhancement. Furthermore, in our study four patients showed an increase in HU measure- ments. These patients had a history of total or partial portal vein thrombosis, which may have altered the liver perfusion. So as primary endpoint of our study, DP-CBCT is a promising technique to evaluate embolisation effect, but further studies are needed for quantitative measurements to foresee the treatment response periprocedurally.

The advantage of seeing the current tumour load on the day of treatment may be beneficial, especially in patients having a time gap between preprocedural cross sectional imaging, MAA angiography, and RE procedures, and it may help prevent misinterpretation of treatment response on the third month post embolisation imaging. One of the reasons for early recurrence after intraarterial treatments is failure to in detect small lesions during embolisation procedures. DSA and single-phase CBCT is inadequate in detection of small nodules [14,18].

The first studies to achieve more than one phase with $\mathrm{CBCT}$ were done with a totally different approach, with injections from two different locations: the hepatic artery injection for arterial phase, and superior mesenteric artery (SMA) injection for portography [7]. Miyayama et al. [9] achieved the two phases by two injections at different time points from the hepatic artery as an alternative to arterioportography and found the sensitivity of lesion detectability to be $88.7 \%$. However, for two separate injections, 30-40 ml contrast media was used for each one. One of the highlights of our study is that it has the advantage of decreasing the amount of contrast media used by $50 \%$ because a single intra-arterial injection was enough to get two phases. The total amount of contrast media used in our study for dual imaging was $9 \mathrm{ml}$, which is also less than is used for cross-sectional CT imaging.

The gathered data resulted in delineating the differences between using early and late arterial phases, having different advantages: EA phases showing hepatic arterial anatomy and LA phases showing better tumour enhancement. In one study it was stated that DP-CBCT was able to change the intra-procedure decision and treatment strategy in $38.6 \%$ of lesions [14]. One study performed with cholangiocellular carcinoma patients showed that lesions missed by digital subtraction angiography (DSA) were either depicted by delayed arterial phase ( $95 \%$ completely and $5 \%$ partially) or early arterial phase (36.6\% completely and $43.9 \%$ partially). Also, lesions missed by early-arterial phase (EAP) were depicted by delayed phase [18]. Another study with metastatic disease showed that missing lesions at early arterial phases were depicted by delayed phases [19]. In our study there was no statistical difference between EAP and LAP in terms of tumour detection because of big tumour burden. But tumour enhancement is found to be better in late arterial phases.

For tumour detection, the acquisition protocol is very important. In the literature, for contrast injection parameters such as injection volume, injection rate, and contrast concentration (diluted/non-diluted), the scan time varies without any standard parameters. We performed two runs 
with two breath holds at $6 \mathrm{~s}$ and $28 \mathrm{~s}$. In some studies the first run was achieved at $3 \mathrm{~s}$. In another study a second run was achieved at $18 \mathrm{~s}$. In a study with HCC patients, two runs were achieved at $4.5 \mathrm{~s}$ and $55 \mathrm{~s}$, respectively, and most of the small tumours were only depicted at EAP [14]. Also, it should be taken into consideration while preparing the protocol that different types of tumours require different protocols.

Our study results are in concordance with prior studies [8], showing that dual-phase imaging with CBCT can achieve enough information for lesion detection. Loffroy et al. [13] showed a sensitivity of $93.9 \%$ with dual-phase imaging compared to MRI in HCC lesions. Another recent study performed with HCC patients who had undergone TACE procedure showed that DP-CBCT tumour detectability was noninferior to CT/MRI [20]. In our study the sensitivity of lesion detection was $100 \%$; however, there were no significant differences in lesion detection compared to cross-sectional imaging, which can be explained because of the more advanced stage of the HCCs in our RE patient group. In general, RE is preferred for patients with more advanced stage HCCs that are not good candidates for TACE, and most of the previously published studies are based on chemoembolisation patient groups, which usually have smaller multifocal lesions and a lower tumour load. Being the first published study on an RE patient group when compared to basal cross-sectional images, acquired DP-CBCT images were at least non-inferior to them, the one patient presenting with only portal vein thrombus being exceptional. When compared to cross-sectional CT, evaluation of non-parenchymal lesions such as portal tumoural thrombus CBCT showed a disadvantage.

As a secondary endpoint of our study, DP-CBCT, improved individualised treatment for each patient by being tumour selective and protective of the normal liver tissue. It allows the interventional radiologist not only to see the tumour burden but also helps in terms of arterial access for selective embolisation and intraprocedural evaluation of success.

There are some limitations to the study. First, the patient population was low for statistical evaluation. Second, the breathing artifacts caused some limitations. To prevent this issue, cooperating patients were included in the study, and lower sedative medication was used. Some studies with one breath hold can be found in the literature. During the procedure we gave two breath hold commands to maintain patient comfort in order to reduce motion artifacts. Based on our measurements, maximum breathing artifacts occurred at the beginning of the first rotation of the C-arm, showing that the patients were not cooperative enough. Other artifacts that affected the image quality resulted from the contrast filling the lumen at the arterial phase. Loffroy et al. and Pellerin et al. used non-diluted contrast medium in their study and stated that it affected the image quality $[13,21]$. We also used diluted contrast media in our study.

Streak artifacts also deteriorated the image quality. Some can be avoided by positioning the patient's arms above the abdomen or by taking the ECG cables off the patient [22]. However, some artifacts such as metallic coils in gastroduodenal and right gastric artery cannot be obviated. Development of software solutions for metal artifacts reduction, similar to the ones used in some multidetector computed tomography (MDCT) technologies, is needed. Despite all these artifacts, dual-phase CT achieved good image quality, at least diagnostically.

Another limitation of our study is that the radiation dose was not calculated. However, in the literature there are studies showing that $\mathrm{CBCT}$ causes lower radiation doses than MDCT $[8,13]$. Also, a new study showed that in the radiation exposure during chemoembolisation, CBCT accounted for only $10 \%$ of the radiation exposure during the whole procedure using standard equipment [23].

\section{Conclusions}

In conclusion, knowing that $\mathrm{CBCT}$ is becoming a part of almost every interventional oncologic procedure, dual-phase imaging may give additional benefits during RE procedures. The future questions that could arise from this study are regarding detection of extrahepatic leakage with DP-CBCT and replacement with hepatic scintigraphy and prediction of survival outcomes of RE, which all need further detailed multi-centric studies.

\section{Conflict of interest}

The authors report no conflict of interest.

\section{References}

1. Bosch FX, Ribes J, Diaz M, et al. Primary liver cancer: worldwide incidence and trends. Gastroenterology 2004; 127: 5-16.

2. Ferlay J, Soerjomataram I, Dikshit RH, et al. Cancer incidence and mortality worldwide: sources, methods and major patterns in GLOBOCAN 2012. Int J Cancer 2015; 136: 359-386.

3. Parkin DM, Whelan SL, Ferlay J, et al. Cancer incidence in five continents. Vol. 8. IARC Sci Publ 2002; 155: 1-781.
4. Bruix J, Sherman M. Practice Guidelines Committee, AASLD. Management of hepatocellular carcinoma. Hepatology 2005; 42: 1208-1236.

5. Mahnken AH, Bruners P, Gunther RW. Techniques of interventional tumor therapy. Deutsches Arzteblatt Int 2008; 105: 646-653.

6. Sangro B, D'Avola D, Iñarrairaegui M, et al. Transarterial therapies for hepatocellular carcinoma. Exp Opin Pharmacother 2011; 12: 1057-1073. 
7. Miyayama S, Matsui O, Yamashiro M, et al. Detection of hepatocellular carcinoma by CT during arterial portography using a cone-beam CT technology: comparison with conventional CTAP. Abdominal imaging 2009; 34: 502-506.

8. Lin MD, Loffroy R, Noordhoek N, et al. Evaluating tumors in transcatheter arterial chemoembolization (TACE) using dual-phase cone-beam CT. Minim Invasiv Ther 2011; 20: 276-281.

9. Miyayama S, Yamashiro M, Okuda M, et al. Detection of corona enhancement of hypervascular hepatocellular carcinoma by $\mathrm{C}$-arm dual-phase cone-beam CT during hepatic arteriography. Cardiovasc Intervent Radiol 2011; 34: 81-86.

10. Peynircioğlu B, Hızal M, Çil B, et al. Quantitative liver tumor blood volume measurements by a C-arm CT post-processing software before and after hepatic arterial embolization therapy: comparison with MDCT perfusion. Diagn Interv Radiol 2015; 21: 71-77.

11. Iwazawa J, Ohue S, Hashimoto N, et al. Survival after C-arm CTassisted chemoembolization of unresectable hepatocellular carcinoma. Eur J Radiol 2012; 81: 3985-3992.

12. Meyer BC, Frericks BB, Voges M, et al. Visualization of hypervascular liver lesions during TACE: comparison of angiographic C-arm CT and MDCT. AJR Am J Roentgenol 2008; 190: 263-269.

13. Loffroy R, Lin MD, Rao P, et al. Comparing the detectability of hepatocellular carcinoma by C-arm dual-phase cone-beam computed tomography during hepatic arteriography with conventional contrast-enhanced magnetic resonance imaging. Cardiovasc Intervent Radiol 2012; 35: 97-104.

14. Wang X, Yarmohammadi H, Cao G, et al. Dual phase cone-beam computed tomography in detecting $<3 \mathrm{~cm}$ hepatocellular carcinomas during transarterial chemoembolization. J Can Res Ther 2017; 13: $38-43$.

15. Wang Z, Chen R, Duran R, et al. Intraprocedural 3D quantification of lipiodol deposition on cone-beam CT predicts tumor response after transarterial chemoembolization in patients with hepatocellular carcinoma. Cardiovasc Intervent Radiol 2015; 38: 1548-1556.
16. Loffroy R, Lin M, Yenokyan G, et al. Intraprocedural C-arm dual-phase cone-beam CT: can it be used to predict short-term response to TACE with drug-eluting beads in patients with hepatocellular carcinoma? Radiology 2013; 266: 636-648.

17. Pellerin O, Shao W, Lin M, et al. Dual phase cone beam CT as a tool to appreciate the micro-embolic effect of radioembolization. Poster presented at ECR 2012. doi: http://dx.doi.org/10.10.1594/ ecr/2012/C-0311.

18. Schernthaner RE, Lin M, Duran R, et al. Delayed phase cone-beam CT improves detectability of intrahepatic cholangiocarcinoma during conventional transarterial chemoembolization. Cardiovasc Intervent Raiol 2015; 38: 929-936.

19. Schernthaner RE, Haroun RR, Duran R, et al. Improved visibility of metastatic disease in the liver during intra-arterial therapy using delayed arterial phase cone-beam CT. Cardiovasc Intervent Radiol 2016; 39: 1429-1437.

20. Yao X, Yan D, Jiang X, et al. Dual-phase cone-beam CT-based navigation imaging significantly enhances tumor detectability and aids superselective transarterial chemoembolization of liver cancer. Acad Radiol 2018; 25: 1031-1037.

21. Pellerin O, Pereira H, Van Ngoc Ty C, et al. Is dual-phase C-arm CBCT sufficiently accurate for the diagnosis of colorectal cancer liver metastasis during liver intra-arterial treatment? Eur Radiol 2019; 29 : 5253-5263.

22. Tognolini A, Louie J, Hwang G, et al. C-arm computed tomography for hepatic interventions: a practical guide. J Vasc Intervent Radiol 2010; 21: 1817-1823.

23. Schernthaner RE, Duran R, Chapiro J, et al. A new angiographic imaging platform reduces radiation exposure for patients with liver cancer treated with transarterial chemoembolization. Eur Radiol 2015; 25: 3255-3262. 